Pacific Journal of Mathematics

ON CERTAIN ALGEBRAIC INTEGERS AND APPROXIMATION
BY RATIONAL FUNCTIONS WITH INTEGRAL COEFFICIENTS 


\title{
ON CERTAIN ALGEBRAIC INTEGERS AND APPROXIMATION BY RATIONAL FUNCTIONS WITH INTEGRAL COEFFICIENTS
}

\author{
David G. CANTOR
}

Let $A$ be a finite set of integers $\left\{a_{1}, a_{2}, \cdots, a_{l}\right\}$ and (possibly) $\infty$. Let $X$ be a nonempty closed subset of $C \cup\{\infty\}$, the field of complex numbers together with $\infty$, under the topology of the Riemann sphere. Suppose that $X$ is symmetric with respect to the field of real numbers $R$ (i.e. if $z \in X$ then $z \in X$ ) and disjoint from $A$. We are interested in the following two problems:

I. Under what conditions do there exist, for each neighborhood $N$ of $X$, infinitely many algebraic numbers $\theta$ such that $1 /\left(\theta-a_{1}\right), 1 /\left(\theta-a_{2}\right), \cdots, 1 /\left(\theta-a_{l}\right)$ are algebraic integers and, if $\infty \in A, \theta$ is itself an algebraic integer, such that all of the (algebraic) conjugates of $\theta$ lie in $N$ ?

II. If $X$ has empty interior and connected complement, then the polynomials are dense in the ring of continuous functions of $X$. What is the uniform closure of the polynomials with integral coefficients in $1 /\left(x-a_{1}\right), 1 /\left(x-a_{2}\right), \cdots$, $1 /\left(x-a_{l}\right)$, and if $\infty \in A, x$ itself?

Problem I was investigated by Raphael Robinson [10]; however instead of requiring the $1 /\left(\theta-a_{i}\right)$ to be algebraic integers, he required that the $b_{i} /\left(\theta-a_{i}\right)$ be algebraic integers, where the $b_{i}$ are integers satisfying $\left(a_{i}-a_{j}\right) \mid b_{i}$ for each $j \neq i$. Our methods are similar to those of Robinson; there are, however, significant differences.

Throughout the remainder of this paper, $A$ will denote a nonempty finite set consisting of real numbers $a_{1}, a_{2}, \cdots, a_{l}$ and (possibly) $\infty$. We assume that $\left|a_{i}-a_{j}\right| \geqq 1$ if $i \neq j$. In $\S \S 2,3,4$, we shall assume that the $a_{i}$ are integers. If $\infty \in A$, we shall sometimes denote it by $a_{0}$. By a symmetric closed (SC) $A$-set $X$, we shall mean a nonempty closed subset of the Riemann sphere, symmetric with respect to the $x$-axis, satisfying $A \cap X=\varnothing$.

If $P(z)$ is a polynomial, we shall denote the leading coefficient of $P(z)$ by $P(\infty)$.

1. Classification of SC $A$-sets. A rational function with real coefficients $\varphi(z)$ is said to be an $A$-function if it is regular except possibly for poles at $a_{i} \in A$. Such a function can be written uniquely in the form $P(z) / D(z)$ where $P(z)$ is a polynomial, $D(z)=\prod_{i=1}^{l}\left(z-a_{i}\right)^{r_{i}}$ where the $r_{i} \geqq 0$ and $P\left(a_{i}\right) \neq 0$ when $r_{i}>0$, for $1 \leqq i \leqq l$. If 
$\infty \notin A$ put $r=\sum_{i=1}^{l} r_{i}$, while if $\infty \in A$, put $r=\max \left(\operatorname{deg} P(z), \sum_{i=1}^{l} r_{i}\right)$ and $r_{0}=r-\sum_{i=1}^{l} r_{i}$. Thus, in either case, $r$ is the number of poles (counting multiplicity) of $\varphi(z)$. We call $\left\{r_{1}, r_{2}, \cdots, r_{l}\right\}$ (or if $\infty \in A,\left\{r_{0}, r_{1}, r_{2}\right.$, $\left.\cdots, r_{l}\right\}$ ) the degree sequence of $\varphi(z)$ (with respect to $A$ ). We shall say that the $A$-function $\varphi(z)$ is an upper $A$-function if all $r_{i}$ are positive and $|P(a)| \geqq 1$ for each $a \in A$. (Recall that by our convention $P(\infty)$ is the leading coefficient of $P(z)$.) We shall say that the $A$-function $\varphi(z)$ is a lower $A$-function if all $r_{i}$ are positive and $0<|P(a)| \leqq 1$ for all $a \in A$. We shall say that the $A$-function $\varphi(z)$ is a normal $A$-function if it is both upper and lower; i.e. if all $r_{i}$ are positive and $|P(a)|=1$ for all $a \in A$. We say that the $A$-function $\varphi(z)$ is an integral $A$-function if $P(z)$ has integral coefficients.

An SC $A$-set $X$ is said to be $A$-small if there exists an upper $A$-function $\varphi(z)$ with $\|\varphi\|_{X}<1$. (Here and throughout \|\|$_{X}$ denotes the sup norm over $X$.) The set $X$ is said to be $A$-large if for each neighborhood $N$ of $X$ there exists a lower $A$-function $\varphi(z)$ satisfying $\{z:|\varphi(z)|=1\} \subset N$ and $X \subset\{z:|\varphi(z)|<1\}$. Note that if $A=\{\infty\}$ then an $A$-small set is simply a set with transfinite diameter $<1$ and an $A$-large set is one with transfinite diameter $\geqq 1$ [3, Theorem I].

THEOREM 1.1. Suppose $A^{\prime}$ is a non-empty subset of $A$. No SC $A$-set $X$ is both A-large and $A^{\prime}$-small.

Proof. Suppose $X$ is both $A$-large and $A^{\prime}$-small. Let $f(z)$ be an upper $A^{\prime}$-function with $\rho=\|f(z)\|_{X}<1$. Choose $\sigma$ satisfying $\rho<\sigma<1$. The set $N_{\sigma}=\{z:|f(z)|<\sigma\}$ is an open neighborhood of $X$. Since $X$ is $A$-large there exists a lower $A$-function $g(z)$ such that $\{z:|g(z)|=1\} \subset N_{\sigma}$. Then, for any $z,|g(z)|=1$ implies $|f(z)|<$ $\sigma<1$. Now suppose that $\infty \in A$; the proof is similar and simpler if $\infty \notin A$. Let $\left\{r_{0}, r_{1}, \cdots, r_{l}\right\}$ be the degree sequence of $f$ (with respect to $A$ ) and let $\left\{s_{0}, s_{1}, \cdots, s_{l}\right\}$ be the degree sequence of $g$. Clearly all $s_{i}$ are $>0$. Choose $h$ so that $r_{h} / s_{h}=\max _{j}\left(r_{j} / s_{j}\right) ; r_{h}$ is $>0$. Put $g_{1}(z)=g(z)^{r_{h}}$ and $f_{1}(z)=f(z)^{s_{h}}$. The degree sequence of $f_{1}$ is $\leqq$ (componentwise) the degree sequence of $g_{1}$, with equality at the $h^{\text {th }}$ component. Put $u(z)=f_{1}(z) / g_{1}(z) ; u(z)$ is regular for all $z$ for which $g_{1}(z) \neq 0$; in particular $u(z)$ is regular in $D=\left\{z:\left|g_{1}(z)\right|>1\right\}$. On $\left|g_{1}(z)\right|=1$, the boundary of $D,\left|f_{1}(z)\right|<\sigma^{s} h<1$, hence $|u(z)|<1$; by the maximum principal this holds for all $z \in D$. But at $z=a_{h} \in D$, $|u(z)| \geqq 1$, since $f_{1}(z)$ is an upper $A^{\prime}$-function and $g_{1}(z)$ is a lower $A$-function. This contradiction completes the proof.

(The author would like to thank the referee for providing this elegant short proof; the original was much longer and more complicated.) We shall need the following. 
Lemma 1.2. Suppose $B=\left(\beta_{i j}\right)$ is a matrix with real entries whose off-diagonal elements are nonnegative. Then either (a) there exists a nonzero vector $x \geqq 0$ such that $B x \geqq 0$, or else (b) $B$ is invertible and $B^{-1}$ is $\leqq 0$.

Proof. Choose $\mu$ so that $B+\mu I$ is $\geqq 0$ and let $\lambda$ be the largest eigenvalue of $B+\mu I$. By an extension of the Perron-Frobenius Theorem [4, Chapter XIII, Theorem 3, p. 66], $-B^{-1}=(\mu I-(B+\mu I))^{-1}$ exists if $\mu>\lambda$ and when that is so is $\geqq 0$, while if $\mu \leqq \lambda$, then $B+\mu I$ has a nonnegative eigenvector $x$ satisfying $(B+\mu I) x=\lambda x$ or $B x=(\lambda-\mu) x \geqq 0$.

The following is closely related to the main result of $\S 4$ of [11].

Theorem 1.3. Let $X$ be an SC A-set. Then either $X$ is $A$ large or there exists a nonempty subset $A^{\prime}$ of $A$ such that $X$ is $A^{\prime}$-small. If $X$ is A-large then for every neighborhood $N$ of $X$ there exists a normal $A$-function $\varphi(z)$ and $R>1$ such that $\{\{z:|\varphi(z)|=$ $R\} \subset N\}$ and $X \subset\{z:|\varphi(z)|\}<R$. Finally, if all finite $a_{i} \in A$ are rational, we may choose $\varphi(z)$ so that its numerator has rational coefficients.

Proof. We shall prove this when $\infty \in A$. The case when $\infty \notin A$ is simpler. The complement of $X$ in the Riemann sphere is a union of components. Let $C_{0}, C_{1}, C_{2}, \cdots, C_{s}$ be those components which have a nonempty intersection with $A$, and suppose they are numbered so that $\infty \in C_{0}$. Put $A_{k}=A \cap C_{k}$ and put $I_{k}=\left\{i: a_{i} \in C_{k}\right\}$. Denote by $X_{k}$ the complement of $C_{k}$ (in the Riemann sphere). Let $N_{k}$ be a neighborhood of $X_{k}$ disjoint from $A$. Suppose that $a_{j} \in A_{k}$. By Theorem $G$ of [3], there exist polynomials $f_{j}(z)$ with real coefficients such that

$$
\begin{aligned}
& \left\{\begin{array}{l}
X_{k} \subset\left\{z:\left|f_{j}\left(1 /\left(z-a_{j}\right)\right)\right|<1\right\} \text { if } j>0 \\
X_{0} \subset\left\{z:\left|f_{0}(z)\right|<1\right\}
\end{array}\right\} \\
& \left\{\begin{array}{l}
\left\{z:\left|f_{j}\left(1 /\left(z-a_{j}\right)\right)\right| \leqq 1\right\} \subset N_{k} \text { if } j>0 \\
\left\{z:\left|f_{0}(z)\right| \leqq 1\right\} \subset N_{0}
\end{array}\right\} .
\end{aligned}
$$

Since each $N_{k} \cap A$ is empty, $\left|f_{0}\left(a_{j}\right)\right|>1$ and $\left|f_{k}\left(1 /\left(a_{j}-a_{k}\right)\right)\right|>1$ for all $k>0$ and $j \neq k$. By replacing each $f_{j}$ by a positive integral power of itself, if necessary, we may assume that the $f_{j}$ all have the same degree, say $d$, and that $d>l$. We are going to construct a function $\varphi(z)$ of the form 
(3)

$$
\begin{aligned}
\varphi(z)= & f_{0}(z)^{t_{0}} \prod_{j \in I_{0}^{\prime}} f_{j}\left(1 /\left(z-a_{j}\right)\right)^{t_{j}} \\
& +\sum_{k=1}^{s} \prod_{j \in I_{k}} f_{j}\left(1 /\left(z-a_{j}\right)\right)^{t_{j}},
\end{aligned}
$$

where here, and throughout this proof, $I_{0}^{\prime}=I_{0}-\{0\}$. We can write $\varphi(z)$ in the form

$$
\varphi(z)=\frac{P(z)}{\prod_{j=1}^{l}\left(z-a_{j}\right)^{d t_{j}}},
$$

where $P(z)$ is a polynomial of degree $d\left(t_{0}+t_{1}+\cdots+t_{l}\right)$, and is explicitly given by

$$
\begin{aligned}
P(z)= & f_{0}(z)^{t_{0}} \prod_{j \in I_{0}^{\prime}}\left[\left(z-a_{j}\right)^{d} f_{j}\left(1 /\left(z-a_{j}\right)\right)\right]^{t_{j}} \cdot \prod_{j \notin I_{0}}\left(z-a_{j}\right)^{d t_{j}} \\
& +\sum_{k=1}^{s} \prod_{j \in I_{k}}\left[\left(z-a_{j}\right)^{d} f_{j}\left(1 /\left(z-a_{j}\right)\right)\right]^{t_{j}} \cdot \prod_{\substack{j \in I_{k} \\
j \neq 0}}\left(z-a_{j}\right)^{d t_{j}} .
\end{aligned}
$$

Then

$$
\begin{aligned}
P(\infty)= & f_{0}(\infty)^{t_{0}} \prod_{j \in I_{0}^{\prime}} f_{j}(0)^{t_{j}}, \\
P\left(a_{i}\right)= & f_{0}\left(a_{i}\right)^{t_{0}} \prod_{\substack{j \in I_{0}^{\prime} \\
j \neq i}}\left[\left(a_{i}-a_{j}\right)^{d} f_{j}\left(1 /\left(a_{i}-a_{j}\right)\right)\right]^{t_{j}} \\
& \times f_{i}(\infty)^{t_{i}} \cdot \prod_{j \notin I_{0}}\left(a_{i}-a_{j}\right)^{d t_{j}} \text { if } i \in I_{0}^{\prime}, \\
P\left(a_{i}\right)= & \prod_{\substack{j \in I_{k} \\
j \neq i}}\left[\left(a_{i}-a_{j}\right)^{d} f_{j}\left(1 /\left(a_{i}-a_{j}\right)\right)\right]^{t_{j}} \\
& \times f_{i}(\infty)^{t_{i}} \cdot \prod_{\substack{j \in I_{k} \\
j \neq 0}}\left(a_{i}-a_{j}\right)^{d t_{j}} \text { if } \quad i \in I_{k}, k \neq 0 .
\end{aligned}
$$

Put

$$
\begin{aligned}
& \beta_{00}=\log \left|f_{0}(\infty)\right|, \\
& \beta_{0 j}=\left\{\begin{array}{ccc}
\log \left|f_{j}(0)\right| & \text { if } & j \in I_{0}^{\prime} \\
0 & \text { if } & j \notin I_{0}
\end{array}\right\}, \\
& \beta_{i 0}=\left\{\begin{array}{ccc}
\log \left|f_{0}\left(a_{i}\right)\right| & \text { if } & i \in I_{0}^{\prime} \\
0 & \text { if } & i \notin I_{0}
\end{array}\right\} ;
\end{aligned}
$$

if $i, j>0$ then put

$$
\beta_{i j}=\left\{\begin{array}{ll}
\log \left|\left(a_{i}-a_{j}\right)^{d} f_{j}\left(1 /\left(a_{i}-a_{j}\right)\right)\right| & \text { if } i \sim j, \quad i \neq j \\
\log \left|f_{j}(\infty)\right| & \text { if } i=j \\
\log \left|\left(a_{i}-a_{j}\right)^{d}\right| & \text { if } i \nsim j
\end{array}\right\} .
$$


Here $i \sim j$ means $i$ and $j$ are in the same $I_{k}$ and $i \nsim j$ means that this is not so. Since $\left|a_{i}-a_{j}\right| \geqq 1$ if $i \neq j, i, j>0$, we see that if $i \neq j$ then $\beta_{i j} \geqq 0$; moreover if $A$ lies in the one component $C_{0}$ and $i \neq j, \beta_{i j}>0$. We have that

$$
\log \left|P\left(a_{i}\right)\right|=\sum_{j=0}^{l} \beta_{i j} t_{j} .
$$

We now apply Lemma 1.2 to the matrix $B=\left(\beta_{i j}\right)$. If case (a) holds, then there exist real $t_{0}, t_{1}, \cdots, t_{l} \geqq 0$, not all 0 , such that all of the sums $\sum_{j} \beta_{i j} t_{j}$ are $\geqq 0$. Let $A^{\prime}$ be the union of those $A_{k}$ for which there exist $j \in I_{k}$ such that $t_{j}>0$. Put $I^{\prime}=\left\{i: a_{i} \in A^{\prime}\right\}$. By replacing each $f_{i}$ by $\lambda f_{i}$, where $\lambda>1$ is small enough that (1) and (2) are still satisfied, we increase $\beta_{i j}$ when $i \sim j$. Hence if $i \in I^{\prime}$, we increase at least one coefficient of a positive $t_{j}$ in the linear form $\sum_{j} \beta_{i j} t_{j}$. Thus we may assume that the linear forms $\sum_{j} \beta_{i j} t_{j}$ are positive when $i \in I^{\prime}$. By modifying the positive $t_{j}$ for which $j \in I^{\prime}$ slightly to make them positive rationals and then multiplying through by a common denominator, we may assume the $t_{j}$ are positive integers, and $\sum_{j} \beta_{i j} t_{j}>0$ when $i \in I^{\prime}$. We can multiply the $t_{j}$ by such a large positive integer that if $i \in I_{k}, i \neq 0$, then $\left|f_{i}\left(1 /\left(z-a_{i}\right)\right)^{t_{i}}\right|$ is $<1 /(s+1)$ for all $z \in X_{k}$ and is $>1$ for all $z$ outside of $N_{k}$. Similarly we will have $\left|f_{0}(z)^{t_{0}}\right|<1 /(s+1)$ for $z \in X_{0}$ and $\left|f_{0}(z)^{t_{0}}\right|>1$ for $z \notin N_{0}$. Now, construct $\varphi$ as in (3) substituting $A^{\prime}$ for $A$ and using the same $N_{k}$ and $f_{j}$. Then $\varphi$ is an upper $A^{\prime}$-function and it is easy to see that $X \subset\{z:|\varphi(z)|<1\}$ so that $X$ is $A^{\prime}$-small.

Next suppose that case (b) of Lemma 1.2 holds. Then $B^{-1} \leqq 0$. Put

$$
t=\left(t_{0}, t_{1}, \cdots, t_{l}\right)^{*}=B^{-1}(-1,-1, \cdots,-1)^{*} .
$$

Then $\sum_{j} \beta_{i j} t_{j}=-1$ and each component $t_{j}$ of $t$ is $>0$, for clearly $t_{j} \geqq 0$ and if $t_{j}=0$, then the $j^{\text {th }}$ row of $B^{-1}$ would be 0 , which is not possible. There is a unique polynomial $g_{0}(z)$ of degree $\leqq l-1$ such that $z^{d}+g_{0}(z)=0$ for $z=a_{1}, a_{2}, \cdots, a_{l}$. Since $d>l$ the polynomial $f_{0}(z)+\delta_{0}\left(z^{d}+g_{0}(z)\right)$ has leading coefficient $f_{0}(\infty)+\delta_{0}$ and takes the same values at $z=a_{1}, a_{2}, \cdots, a_{l}$ as $f_{0}(z)$. Thus replacing $f_{0}(z)$ by $f_{0}(z)+\delta_{0}\left(z^{d}+g_{0}(z)\right)$ would change $\beta_{00}$ but none of the other $\beta_{i 0}$. If $\delta_{0}$ is small enough then (1) and (2) would remain satisfied. Similar comments apply to $f_{1}, f_{2}, \cdots, f_{l}$. Thus there exists $\varepsilon>0$ such that each $f_{j}$ can be modified in such a way that $\beta_{i j}$ is unchanged if $i \neq j$, while $\beta_{j j}$ varies over an interval of length $2 \varepsilon$, and at the same time (1) and (2) remain valid. Choose positive rational $t_{j}^{\prime}$ so close to $t_{j}, 0 \leqq j \leqq l$, that $\left|\sum_{i=0}^{l} \beta_{i j}\left(t_{j}-t_{j}^{\prime}\right) / t_{i}^{\prime}\right|<\varepsilon$ for $0 \leqq i \leqq l$.

Now put $\beta_{i j}^{\prime}=\beta_{i j}$ if $i \neq j$ and choose $\beta_{i i}^{\prime}$ so that $\sum_{j=0}^{l} \beta_{i j}^{\prime} t_{j}^{\prime}=-1$ 
for $0 \leqq i \leqq l$. Then $\left|\beta_{i i}^{\prime}-\beta_{i i}\right|=\left|\sum_{j=0}^{l} \beta_{i j}\left(t_{j}-t_{j}^{\prime}\right) / t_{i}^{\prime}\right|<\varepsilon$ for $0 \leqq i \leqq l$. Now modify the $f_{i}$ slightly so that the $\beta_{i j}$ are replaced by the $\beta_{i j}^{\prime}$ and the $t_{i}$ by the $t_{i}^{\prime}$, still preserving (1) and (2). Thus, after this replacement we may assume that the $t_{i}$ are all positive rational numbers. Now multiply the $t_{j}$ by such a large positive integer $n$ that they become integers and such that if $i \in I_{k}, i \neq 0$, then $\left|f_{i}\left(1 /\left(z-a_{i}\right)\right)^{t_{i}}\right|$ is $<1 /(s+1)$ for $z \in X_{k}$ and $>2$ for $z \notin N_{k}$. Similarly $\left|f_{0}(z)\right|^{t_{0}}$ is $<1 /(s+1)$ for $z \in X_{0}$ and $>2$ for $z \notin N_{0}$. Then $\varphi$ as defined in (3) is a lower $A$-function and all of the $\left|P\left(a_{j}\right)\right|$ are equal to $1 / e^{n}$. By replacing $\varphi$ by $\varphi^{2}$, we obtain a lower $A$-function $\varphi$ with $P\left(a_{j}\right)=1 / e^{2 n}$ for $0 \leqq j \leqq l$. For $z \notin N_{k}$ all but one of the terms in (3) have absolute value $<1 /(s+1)$ while the remaining term has value $>2$. Thus $|\varphi(z)|$ is $>1$ outside of each $N_{k}$. If $z \in X$, however, then each term in (3) has absolute value $<1 /(s+1)$ and $|\varphi(z)|<1$. Let $Y$ be the union of $X$ and those components of the complement of $X$ which are disjoint from $A$; i.e., $Y$ is obtained from $X$ by filling in those holes which contain no $\alpha_{i}$. If $N$ is any neighborhood of $X$, then there exist neighborhoods $N_{k}$ of $X_{k}$ such that $\bigcap_{k} N_{k} \subset N \cup Y$. The $\varphi$, as modified above and corresponding to this choice of the $N_{k}$ is lower, $X \subset\{z:|\varphi(z)|<1\}$, and $\{z:|\varphi(z)|=1\} \subset N$. Thus $X$ is $A$-large. Then $\varphi_{1}(z)=\varphi(z) e^{2 n}$ is $A$-normal and $X \subset$ $\left\{z:\left|\varphi_{1}(z)\right|<e^{2 n}\right\}$ and $\left\{z:\left|\varphi_{1}(z)\right|=e^{2 n}\right\} \subset N$.

Finally, suppose the $\alpha_{i}$ are rational, and $\varphi_{1}(z)=P_{1}(z) / D(z)$; then $P_{1}\left(a_{i}\right)=1,0 \leqq i \leqq l$. We can choose a polynomial $C(z)$ of degree $<\operatorname{deg}\left(P_{1}(z)\right)-l$ and with arbitrarily small coefficients such that $P_{2}(z)=P_{1}(z)+C(z) \prod_{i=1}^{l}\left(z-a_{\imath}\right)$ has all coefficients of terms of degree $\geqq l$ rational. Since $P_{2}\left(a_{i}\right)=1,0 \leqq i \leqq l$, the remaining coefficients are rational. If $C(z)$ is small enough then $\varphi_{2}(z)=P_{2}(z) / D(z)$ meets the requirements of the theorem.

REMARK 1.4. The $A^{\prime}$ in the above theorem is a union of some of the $A_{k}$. In particular if all $a_{i}$ lie in one component of the complement of $X$, then either $X$ is $A$-large or $X$ is $A$-small.

We shall need the following theorem in $\S 4$.

Theorem 1.5. A finite SC A-set $X$ is A-small.

Proof. By standard interpolation theory results, there exists a monic polynomial $P$ which vanishes at each element of $X$ and is 1 at each finite element of $A$. We may choose $P$ to have degree $\geqq l+1$, and then $\varphi(z)=P(z) / \prod_{i=l}^{l}\left(z-a_{i}\right)$ is a normal $A$-function which has absolute value $<1$ on $X$. 
THEOREM 1.6. If $X$ is an A-small set, then there exists a normal $A$-function $\varphi(z)$ such that $\|\varphi(z)\|_{X}<1$.

Proof. We shall prove this in the case when $\infty \in A$. The case when $\infty \notin A$ is simpler. By definition there exists an upper $A$-function $Q(z) / D(z)$ such that $\|Q(z) / D(z)\|_{x}<1$. Suppose $D(z)=\prod_{j=1}^{l}\left(z-a_{j}\right)^{r_{j}}$. Since $z$ and each of the functions $1 /\left(z-a_{j}\right)$ is bounded on $X$, there exists an integer $n \geqq 1$ so large that $\left\|Q(z)^{n} / D(z)^{n-1}\right\|_{X}<1 /(l+1)$ and $\left\|Q(z)^{n} /\left(\left(z-a_{j}\right)^{d_{j}} D(z)^{n-1}\right)\right\|_{x}<1 /(l+1)$ for $1 \leqq j \leqq l$. Now put

$$
P(z)=\left(\alpha_{0} D(z)+\sum_{j=1}^{l} \alpha_{j} D(z) /\left(z-a_{j}\right)^{r_{j}}\right) Q(z)^{n}
$$

where the $\alpha_{i}$ will be chosen later. Then $P(\infty)=\alpha_{0} Q(\infty)^{n}$ and $P\left(a_{i}\right)=$ $\alpha_{i} \prod_{j \neq i}\left(a_{i}-a_{j}\right)^{r_{j}} Q\left(a_{i}\right)^{n}$. Thus there exist unique choices for the $a_{i}$ so that $P(\infty)=1$ and all $P\left(a_{i}\right)=1$, and the $\alpha_{i}$ will have absolute value $\leqq 1$. Put $\varphi(z)=P(z) / D(z)^{n} ; \varphi(z)$ is a normal $A$-function and

$$
\begin{aligned}
\|\varphi(z)\|_{X} \leqq & \left|\alpha_{0}\right| \cdot\left\|Q(z)^{n} / D(z)^{n-1}\right\|_{X} \\
& +\sum_{j=1}^{l}\left|\alpha_{j}\right| \cdot\left\|Q(z)^{n} /\left(\left(z-a_{j}\right)^{r} j D(z)^{n-1}\right)\right\|_{X} \\
< & 1 .
\end{aligned}
$$

If $N(z)$ is a nonconstant polynomial, then any power series $u(z)$ can be written uniquely in the form

$$
u(z)=\sum_{i=0}^{\infty} c_{i}(z) N(z)^{2}
$$

where the $c_{i}(z)$ are polynomials of degree $<\operatorname{deg}(N(z))$. This is the special case, $A=\{\infty\}$, of the next lemma. To extend to general sets $A$, we must replace $N(z)$ by a rational function which has poles at each $a_{i} \in A$, and allow the $c_{2}(z)$ to be rational functions with poles of bounded order at each $a_{i} \in A$. In the following lemma, $N(z)$ is replaced by $N(z) / D(z)$ and the $c_{i}(z)$ by the $c_{i}(z) / D(z)$.

LEMma 1.7. Suppose $\infty \in A$ and $D(z)=\prod_{i=1}^{l}\left(z-a_{i}\right)^{r_{i}}$ where the $r_{i}$ are $>0$. Suppose $N(z)$ is a polynomial, relatively prime to $D(z)$, of degree $r=\sum_{i=0}^{l} r_{i}$ where $r_{0}$ is $>0$. If $u(z)$ is an A-function satisfying $u\left(a_{i}\right) \neq 0,0 \leqq i \leqq r$, we can write uniquely

$$
u(z)=\sum_{i=0}^{n} c_{i}(z) N(z)^{i} / D(z)^{i+1}
$$

where $n$ is the least integer $\geqq 0$ such that

$$
-\operatorname{ord}_{\infty} u(z) \leqq(n+1) r_{0}-1
$$


and

$$
-\operatorname{ord}_{a_{i}} u(z) \leqq(n+1) r_{i} \text { for } 1 \leqq i \leqq l ;
$$

and where the $c_{\imath}(z)$ are polynomials of degree $<r$ and $c_{n}(z)$ is not 0 .

Suppose $\infty \notin A$ and $D(z)=\prod_{i=1}^{l}\left(z-a_{i}\right)^{r_{i}}$, where the $r_{i}$ are $>0$. Suppose $N(z)$ is a polynomial, relatively prime to $D(z)$ and of degree $\leqq r=\sum_{i=1}^{l} r_{i}$. If $u(z)$ is an A-function satisfying $u\left(a_{i}\right) \neq 0$, $1 \leqq i \leqq r$ and vanishing at $\infty$, we can write, uniquely,

$$
u(z)=\sum_{i=0}^{n} c_{i}(z) N(z)^{i} / D(z)^{i+1}
$$

where $n$ is the least integer $\geqq 0$ such that

$$
-\operatorname{ord}_{a_{i}} u(z) \leqq(n+1) r_{i} \text { for } 1 \leqq i \leqq l ;
$$

and where the $c_{i}(z)$ are polynomials of degree $<r$, and $c_{n}(z)$ is not 0 .

Proof. We give the proof for the case $\infty \in A$, and it is by induction on $n$. The result is clear when $n=0$, for then $D(z) u(z)$ is a polynomial of degree $<r$. If $n \geqq 1$, choose the polynomial $c_{0}(z)$ of degree $<r$ and $\equiv u(z) D(z)(\bmod N(z))$; then the polynomial $D(z)^{n}\left(u(z) D(z)-c_{0}(z)\right)$ is divisible by $N(z)$. Note that this is the unique choice for $c_{0}(z)$. Then

$$
-\operatorname{ord}_{\infty}\left(u(z) D(z)-c_{0}(z)\right) / N(z) \leqq n r_{0}-1
$$

and

$$
-\operatorname{ord}_{a_{i}}\left(u(z) D(z)-c_{0}(z)\right) / N(z) \leqq n r_{i}
$$

for $1 \leqq i \leqq l$. Thus, inductively, we have, uniquely,

$$
\left(u(z)-c_{0}(z) / D(z)\right) \frac{D(z)}{N(z)}=\sum_{i=0}^{n-1} c_{i+1}(z) \frac{N(z)^{i}}{D(z)^{i+1}}
$$

and then

$$
u(z)=\sum_{i=0}^{n} c_{i}(z) N(z)^{i} / D(z)^{i+1}
$$

LEMma 1.8. Suppose $\infty \in A$ and $X$ is an SC A-set. Suppose $g(z)=P(z) / D(z)$ is a normal A-function where $D(z)=\prod_{i=1}^{l}\left(z-a_{i}\right)^{r_{i}}$ and $P(z)$ has degree $r=\sum_{i=0}^{l} r_{i}$. Put $\lambda=\|g(z)\|_{X}^{1 / r}$. Then there exists $M>0$ and for each integer $n \geqq 0$ an A-function $\theta_{n}(z)$ such that when $\theta_{n}(z)$ is expanded according to Lemma 1.7, with $N(z)=$ $z^{r_{0}} D(z)+1$, 


$$
\theta_{n}(z)=\sum_{i=0}^{s} d_{i}(z) N(z)^{i} / D(z)^{i+1},
$$

then $n=r s+t$, where $0 \leqq t<r, d_{s}(z)$ is a monic polynomial of degree $t,\left\|\theta_{n}(z)\right\|_{X}<M \lambda^{n}$, and $\left|\theta_{n}(z)\right|<M|g(z)|^{1+n / r}$ when $|g(z)|>1$.

Suppose $\infty \notin A$ and $X$ is an SC A-set. Suppose $g(z)=P(z) / D(z)$ is a normal A-function where $D(z)=\prod_{i=1}^{l}\left(z-a_{i}\right)^{r_{i}}$ and $P(z)$ has degree $<r=\sum_{i=1}^{l} r_{i}$. Put $\lambda=\|g(z)\|_{X}^{1 / r}$. Then there exists $M>0$ and for each integer $n \geqq 0$ an A-function $\theta_{n}(z)$ such that when $\theta_{n}(z)$ is expanded according to Lemma 1.7, using $N(z)=1$,

$$
\theta_{n}(z)=\sum_{i=0}^{s} d_{i}(z) / D(z)^{i+1},
$$

then $n=r s+t$ where $0 \leqq t<r$ and $d_{s}(z)$ is a monic polynomial of degree $t,\left\|\theta_{n}(z)\right\|_{X}<M \lambda^{n}$, and $\left|\theta_{n}(z)\right|<M|g(z)|^{1+n / r}$ when $|g(z)|>1$.

Proof. Suppose first that $\infty \in A$. Expand $g(z)^{m}$ by Lemma 1.7:

$$
g(z)^{m}=\sum_{i=0}^{m} c_{i}(z) N(z)^{i} / D(z)^{i+1} .
$$

It is easy to verify that $c_{m}(z)=D(z)$, hence is monic of degree $\sum_{l=1}^{l} r_{i}$. Then $g(z)^{m}$ will serve for $\theta_{(m+1) r-r_{0}}$. The functions

$$
z \theta_{(m+1) r-r_{0}}, z^{2} \theta_{(m+1) r-r_{0}}, \cdots, z^{r_{0}-1} \theta_{(m+1) r-r_{0}}
$$

will serve for $\theta_{(m+1) r-r_{0}+1}, \theta_{(m+1) r-r_{0}+2}, \cdots, \theta_{(m+1) r-1}$, respectively. The functions $\theta_{(m+1) r-r_{0}} /\left(z-a_{i}\right), \theta_{(m+1) r-r_{0}} /\left(z-a_{i}\right)^{2}, \cdots, \theta_{(m+1) r-r_{0}}(z) /\left(z-a_{i}\right)^{r_{1}}$ will serve for $\theta_{(m+1) r-r_{0}-1}, \theta_{(m+1) r-r_{0}-2}, \cdots, \theta_{(m+1) r-r_{0}-r_{1}}$, respectively. Continuing in this way, dividing next by $\left(z-a_{1}\right)^{r_{1}}\left(z-a_{2}\right)$, then $\left(z-a_{1}\right)^{r_{1}}\left(z-a_{2}\right)^{2}, \cdots$, and so forth will give the remaining functions. Since all of the functions $z, 1 /\left(z-a_{1}\right), \cdots, 1 /\left(z-a_{l}\right)$ are bounded on $X$ and $z^{r_{0}} / g(z), 1 /\left(\left(z-a_{1}\right)^{r_{1}} g(z)\right), \cdots, 1 /\left(\left(z-a_{l}\right)^{r_{l}} g(z)\right)$ are bounded when $|g(z)|>1$, there exists $M>0$ as required for the Lemma. If $\infty \notin A$, use the above procedure with $r_{0}=0$, omitting $z$ and $z^{r_{0}} / g(z)$ when defining $M$.

2. Classification of $A$-sets-Integral $A$. In this and succeeding sections we assume that the $a_{\imath} \in A$ are integers and strengthen the results of $\S 1$.

Theorem 2.1. If $X$ is A-small there exists an integral, normal $A$-function $\varphi(z)$ such that $\|\varphi(z)\|_{X}<1$.

Proof. We give the proof in the case that $\infty \in A$. There exists an $A$-normal function $P(z) / D(z)$, where $D(z)=\prod_{\imath=1}^{l}\left(z-a_{i}\right)^{r_{i}}$ and $\|P(z) / D(z)\|_{X}<1$. Suppose $P(z)$ has degree $r=\sum_{i=0}^{l} r_{i}$ and put 
$N(z)=z^{r_{0}} D(z)+1$. Choose $m>0$. For any $n>m$, the function $(P(z) / D(z))^{n}\left(1+1 /\left(\left(z-a_{1}\right)\left(z-a_{2}\right) \cdots\left(z-a_{l}\right)\right)\right)$ is $A$-normal and by Lemma 1.7 can be written in the form $\sum_{i=0}^{n} c_{i}^{(n)}(z) N(z)^{i} / D(z)^{i+1}$. It is easy to verify that $c_{n}^{(n)}(z)=D(z)\left(1+1 /\left(\left(z-a_{1}\right)\left(z-a_{2}\right) \cdots\left(z-a_{l}\right)\right)\right)$. We can successively add $\varepsilon_{1} \theta_{n r-1}(z), \varepsilon_{2} \theta_{n r-2}(z), \cdots, \varepsilon_{n r-m r} \theta_{m r}(z)$, where the $\theta_{i}(z)$ are the functions defined in Lemma 1.8 and the $\varepsilon_{i}$ are real numbers in the interval $[-1 / 2,1 / 2)$, so as to obtain a function

$$
h_{n}(z)=\sum_{i=0}^{n} d_{i}^{(n)}(z) N(z)^{i} / D(z)^{i+1}
$$

where $d_{n}^{(n)}(z)=c_{n}^{(n)}(z)$ and $d_{m}^{(n)}(z), \quad d_{m+1}^{(n)}(z), \cdots, d_{n-1}^{(n)}(z)$ have integral coefficients. Furthermore, with $M$ and $\lambda$ as defined in Lemma 1.8,

$$
\left\|h_{n}(z)\right\|_{X}<M^{\prime}\|P(z) / D(z)\|_{X}^{n}+M\left(\lambda^{m r}+\lambda^{m r+1}+\cdots+\lambda^{n r-1}\right)<M^{\prime \prime} \lambda^{m r}
$$

where $M^{\prime}=\left\|\left(1+1 /\left(\left(z-a_{1}\right)\left(z-a_{2}\right) \cdots\left(z-a_{l}\right)\right)\right)\right\|_{X}$ and $M^{\prime \prime}=\max \left(M, M^{\prime}\right)$ $/(1-\lambda)$. We can choose $m$ so large that $M^{\prime \prime} \lambda^{m r}<1 / 3$. For each $n>m$, we obtain such a function $h_{n}(z)$ and in the expansion (7), all of the $d_{i}^{(n)}(z)$, except those with $i<m$, have integral coefficients. We can find $n_{2}>n_{1}>m$ so that all of the coefficients of the $d_{i}^{\left(n_{2}\right)}(z)-$ $d_{i}^{\left(n_{1}\right)}(z)$, for $0 \leqq i \leqq m-1$, are extremely small modulo 1 .

When this is the case put $\varphi(z)=\sum_{l=0}^{n_{2}} e_{i}(z) N(z)^{i} / D(z)^{i+1}$ where $e_{i}(z)$ is the polynomial with integral coefficients nearest to $d_{i}^{\left(n_{2}\right)}(z)-$ $d_{i}^{\left(n_{1}\right)}(z)$; here we put $d_{2}^{\left(n_{1}\right)}(z)=0$ when $i>n_{1}$. If $n_{1}$ and $n_{2}$ were chosen appropriately, $\varphi(z)$ will satisfy $\|\varphi(z)\|_{X}<1$ and since $e_{n_{2}}(z)=$ $\left(1+1 /\left(\left(z-a_{1}\right)\left(z-a_{2}\right) \cdots\left(z-a_{l}\right)\right)\right) D(z), \varphi(z)$ is normal.

THEOREM 2.2. Suppose $X$ is A-large. Then for each neighborhood $N$ of $X$ there exists an integral normal $A$-function $\varphi(z)$ and an integer $S>1$ such that $\{z:|\varphi(z)|=S\} \subset N$ and $X \subset\{z:|\varphi(z)|<S\}$.

Proof. We give the proof for the case that $\infty \in A$. By Theorem 1.3, there exists a normal $A$-function $g(z)$ with rational coefficients and $R>1$ such that $\{z:|g(z)|=R\} \subset N$ and $X \subset\{z:|g(z)|<R\}$. We can write $g(z)=N(z) / D(z)+c(z) /(h D(z))$ where, as usual, $D(z)=$ $\prod_{i=1}^{l}\left(z-a_{i}\right)^{r_{i}}, \quad N(z)=z^{r_{0}} D(z)+1, r=\sum_{i=0}^{l} r_{i}, c(z)$ is a polynomial of degree $<r$ with integral coefficients satisfying $c\left(a_{i}\right)=0$ for $i \leqq$ $i \leqq l$, and $h$ is a positive integer. We can write

$$
\begin{aligned}
g(z)^{n}= & (N(z)+c(z) / h)^{n} / D(z)^{n} \\
= & \sum_{i=0}^{m-1} N(z)^{n-i} c(z)^{i}\left(\begin{array}{c}
n \\
i
\end{array}\right) /\left(h^{i} D(z)^{n}\right) \\
& +\sum_{i=m}^{n} N(z)^{n-i} c(z)^{i}\left(\begin{array}{c}
n \\
i
\end{array}\right) /\left(h^{i} D(z)^{n}\right),
\end{aligned}
$$


where $m<n$ will be chosen later in this proof.

When the first sum is written as a rational function in $z$ with denominator $D(z)^{n}$, each coefficient of a power of $z$ in the numerator will be a polynomial in $n$ with rational coefficients. Since the polynomial $\left(\begin{array}{l}n \\ i\end{array}\right)$ in $n$ is divisible by $n$ for each $i>0$, the numerator polynomial will have integral coefficients when $n$ is divisible by a certain fixed integer $n_{0}$.

Since $c(z)$ has degree $<r$, the second sum has a pole at $\infty$ of order $\leqq(n-m) r+m(r-1)-n\left(r-r_{0}\right)=n r_{0}-m$. Since $c(z)$ vanishes at each $a_{i}$, the second sum has a pole at $a_{i}$ of order $\leqq n r_{i}-m$. By Lemma 1.7, the second sum can be written in the form

$$
\sum_{i=0}^{k} b_{i}(z) N(z)^{i} / D(z)^{i+1}
$$

where $k$ is the least integer $\geqq 0$ satisfying

$$
\begin{aligned}
& k+1 \geqq n-(m-1) / r_{0} \\
& k+1 \geqq n-m / r_{i} ; \quad 1 \leqq i \leqq l .
\end{aligned}
$$

Put $j=(k+1) r-1$. Let $\theta_{0}(z), \theta_{1}(z), \theta_{2}(z), \cdots$ be the functions constructed in Lemma 1.8 using $P(z) / D(z)=g(z)$. By adding successively $\varepsilon_{j} \theta_{j}(z), \quad \varepsilon_{j-1} \theta_{j-1}(z), \cdots$ where the $\varepsilon_{i}$ are chosen appropriately from the interval $[-1 / 2,1 / 2)$, to $g(z)^{n}$ we obtain an integral normal $A$-function $f_{n}(z)$. Choose $R_{1}$ and $R_{2}$ close to $R$ with $1<R_{1}<R<R_{2}$ such that $X \subset\left\{z:|g(z)|<R_{1}\right\}$ and $\left\{z: R_{1} \leqq|g(z)| \leqq R_{2}\right\} \subset N$. Then $f_{n}(z)$ differs from $g(z)^{n}$ in the set $\left\{z: R_{1}<|g(z)|\right\}$ by less than $M|g(z)|\left(|g(z)|^{j / r}+|g(z)|^{(j-1) / r}+\cdots+1\right)$ or by less than $M^{\prime}|g(z)|^{2+j / r}$ where $M^{\prime}=M /\left(R_{1}^{1 / r}-1\right)$. Hence if $n / j$ is large enough, $f_{n}(z)$ does not vanish when $\left|g_{n}(z)\right| \geqq R_{2}$. Similarly, if $|g(z)| \leqq R_{1}$, then $f_{n}(z)$ differs from $g(z)^{n}$ by $\leqq M^{\prime \prime} R_{1}^{j ! r}$. Thus by the maximal principal, if $|g(z)| \geqq R_{2},\left|f_{n}(z)\right| \geqq(1-\delta) R_{2}^{n}$ and if $|g(z)| \leqq R_{1},\left|f_{n}(z)\right| \leqq(1+\delta) R_{1}^{n}$, where $\delta>0$ can be made arbitrarily close to 0 by choosing $m$ large. If $n$ is large enough and divisible by $n_{0}$ there will be an integer $S$ in the interval $\left((1+\delta) R_{1}^{n},(1-\delta) R_{2}^{n}\right)$; putting $\varphi(z)=f_{n}(z)$ completes the proof.

3. A-integers. An algebraic number $\theta$ is said to be an $A$-integer if $1 /\left(\theta-a_{i}\right)$ is an algebraic integer for each $a_{i} \in A$ and $\theta$ is an algebraic integer if $\infty \in A$.

LEMMA 3.1. If $\varphi(z)=P(z) / D(z)$ is an integral normal A-function and $\theta$ is a complex number such that $\varphi(\theta)=\alpha$ is an algebraic 
integer, then $\theta$ is an A-integer.

Proof. The polynomial $P(z)-\alpha D(z)$ has algebraic integer coefficients and is satisfied by $\theta$. If $\infty \in A$, then this polynomial is monic of degree $r$ and hence $\theta$ is an algebraic integer. Since $P\left(a_{i}\right)$ $\alpha D\left(a_{i}\right)=P\left(a_{i}\right)=1$, the polynomial with algebraic integer coefficients satisfied by $1 /\left(\theta-a_{i}\right)$ is monic and $1 /\left(\theta-a_{i}\right)$ is an algebraic integer.

LEMma 3.2. If $\varphi(z)$ is an integral A-function and $\theta$ is an $A$-integer then $\varphi(\theta)$ is an algebraic integer.

Proof. We first show that the ring generated by the functions $1,1 /\left(z-a_{1}\right), 1 /\left(z-a_{2}\right), \cdots, 1 /\left(z-a_{l}\right)$, and if $\infty \in A$, the function $z$, contains all integral $A$-functions. This is clear if $\infty \in A$, so suppose $\infty \notin A$. Suppose $P(z) / D(z)$ is an integral $A$-function, $D(z)=\sum_{i=1}^{l}$ $\left(z-a_{\imath}\right)^{r_{i}}$, and $r=\sum_{l=1}^{l} r_{i}$. We proceed by induction on $r$. If $r=0$, the result is clear. Otherwise some $r_{i}$, say $r_{1}$, is $>0$. Then $P(z) / D(z)=\left(P(z)-P\left(a_{1}\right)\right) / D(z)+P\left(a_{1}\right) / D(z)$. Clearly $P\left(a_{1}\right) / D(z)$ is in the ring and since $\left(z-a_{1}\right) \mid\left(P(z)-P\left(a_{1}\right)\right),\left(P(z)-P\left(a_{1}\right)\right) / D(z)$ is in the ring by induction. Since each $1 /\left(\theta-a_{i}\right)$ is an algebraic integer and if $\infty \in A, \theta$ is an algebraic integer, $\varphi(\theta)$ is an algebraic integer.

We now give the basic results of this section.

THEOREM 3.3. Let $X$ be a set which is not A-large. Then there exists a neighborhood of $X$ which contains only finitely many complete conjugate sets of A-integers.

Proof. By Theorems 1.3 and 2.1, $A$ contains a nonempty subset $A^{\prime}$ for which there exists an integral $A^{\prime}$-function $\varphi(z)$ such that $\|\varphi(z)\|_{X}<1$. Pnt $N=\{z:|\varphi(z)|<1\}$. If $\left\{\theta_{1}, \theta_{2}, \cdots, \theta_{m}\right\}$ is a complete conjugate set of $A$-integers contained in $N$, then $\left\{\varphi\left(\theta_{1}\right), \varphi\left(\theta_{2}\right), \cdots\right.$, $\left.\varphi\left(\theta_{m}\right)\right\}$ is a sequence of algebraic integers, consisting of repetitions of a complete conjugate set. Since each $\varphi\left(\theta_{i}\right)$ has absolute value $<1$, the norm of each is $<1$, hence 0 . Thus each $\varphi\left(\theta_{i}\right)=0$ and so the total number of $\theta_{i}$ is $\leqq r$, the degree of the numerator of $\varphi(z)$.

THEOREM 3.4. Let $X$ be an A-large set. Then every neighborhood $N$ of $X$ contains infinitely many complete sets of conjugate A-integers.

Proof. Let $N$ be a neighborhood of $X$. By Theorem 2.2 there exists an integral normal $A$-function $\varphi(z)$ and an integer $S>1$ such that $\{z:|\varphi(z)|=S\} \subset N$. The solutions to $\varphi(z)^{n}=S^{n}$ lie in $N$ and 
by Lemma 3.1 are $A$-integers.

It is probable that if $X$ is an $A$-large subset of $\boldsymbol{R}$ then every real neighborhood of $X$ contains infinitely many complete sets of conjugate $A$-integers. In the case $A=\{\infty\}$ and $X$ is a finite union of closed intervals in $\boldsymbol{R}$ this was shown by Robinson in [7] and [8], and in the case $X$ is a closed interval and $A=\{\infty, 0\}$ this was shown by Robinson in [9].

4. Approximation. Let $X$ be an SC set with empty interior and such that each component of the complement of $X$ in $C$ contains an element of $A$. A complex valued function $f$ on $X$ is called symmetric if $f(\bar{x})=\overline{f(x)}$ for all $x \in X$. We shall denote the ring of continuous symmetric functions on $X$ by $C_{s}(X)$. A theorem of Mergelyan [6, Theorem 2.3] asserts that the $A$-functions are dense, in the uniform norm, in $C_{s}(X)$. We are interested in investigating the uniform closure of the integral $A$-functions in $C_{s}(X)$. For the case $A=\{\infty\}$ see [1] and [5]. If $Y$ is an SC subset of $X$, we shall say that the symmetric function $f$ is matchable on $Y$ if there exists an integral $A$-function $p$ such that $p(y)=f(y)$ for all $y \in Y$ and we shall say that $f$ is approximable on $Y$ if for each $\varepsilon>0$ there exists an integral $A$-function $p$ such that $\|p-f\|_{Y}<\varepsilon$.

Theorem 4.1. If $X$ is A-large then the integral A-functions form a closed discrete subset of $C_{s}(X)$.

Proof. Suppose $\varphi_{1}$ and $\varphi_{2}$ are integral $A$-functions with $\left\|\varphi_{1}-\varphi_{2}\right\|_{X}<1$. If $\varphi_{1} \neq \varphi_{2}$ then $\varphi_{1}-\varphi_{2}$ is an upper $A^{\prime}$-function for some nonempty $A^{\prime} \subset A$. But this implies that $X$ is $A^{\prime}$-small, contradicting Theorem 1.1.

Now define $J(X, A)$ to be the union of the complete sets of conjugate $A$-integers contained in $X$. Note that if $X$ is not $A$-large then, by Theorem $3.3, J(X, A)$ is finite.

THEOREM 4.2. If $X$ is $A^{\prime}$-small for some non-empty $A^{\prime} \subset A$ and each component of the complement of $X$ contains an element of $A^{\prime}$ then $f \in C_{s}(X)$ is approximable on $X$ if and only if it is matchable on $J(X, A)$.

Proof. First observe that if $\phi$ is an integral $A$-function which satisfies $|\varphi(x)|<1$ for each $x \in J(X, A)$, then $\phi(x)=0$ for each $x \in J(X, A)$. Indeed $J(X, A)$ is the disjoint union of complete sets of conjugate $A$-integers. Let $x_{1}, x_{2}, \cdots, x_{r}$ be one such complete set. 
Then $\prod_{i=1}^{r} \varphi\left(x_{i}\right)$ is a rational integer with absolute value $<1$. Hence the product is 0 , and so at least one of the $\varphi\left(x_{i}\right)=0$, and since they are conjugate they are all 0 , and $\varphi$ vanishes on $J(X, A)$. Now suppose $f$ is approximable on $X$ and that $\left\|p_{1}-f\right\|_{x}<1 / 2$ and $\left\|p_{2}-f\right\|_{X}<1 / 2$. Then $\left\|p_{1}-p_{2}\right\|_{X}<1$. By what we proved above $p_{1}(x)=p_{2}(x)$ for all $x \in J(X, A)$. Since $\left\|p_{2}-f\right\|_{X}$ can be chosen arbitrarily small, it follows that $f(x)=p_{1}(x)$ for all $x J(X, A)$; hence that $f$ is matchable on $J(X, A)$.

Assume $\infty \in A^{\prime}$. The proof is similar when $\infty \notin A^{\prime}$. Since $X$ is $A^{\prime}$-small, there exists a normal integral $A^{\prime}$-function $\varphi$ with $\|\varphi\|_{X}<1$. Let $K$ be the (finite) set of those zeros of $\varphi$ contained in $X$. Since $\|\varphi\|_{X}<1,|\varphi(x)|<1$ for all $x \in J(X, A)$ and hence $\varphi$ vanishes on $J(X, A)$. Thus $J(X, A) \subset K$.

Let $m$ be a positive integer. By a standard extension of the Stone-Weierstrass theorem, the closed ideal generated by $\varphi^{m}$ in $C_{s}(X)$ consists of all functions $g \in C_{s}(X)$ vanishing on $K$. By our assumption about $X$, the $A^{\prime}$-functions are dense in $C_{s}(X)$. Thus if $\varepsilon>0$ and $g \in C_{s}(X)$ vanishes on $K$, there exists an $A^{\prime}$-function $h(x)$ such that $\left\|\varphi(x)^{m} h(x)-g(x)\right\|_{X}<\varepsilon$. By Lemma 1.7, we can write

$$
\varphi(x)^{m} h(x)=\sum_{j=m}^{n}\left(h_{i}(x) / D(x)\right) \varphi(x)^{j},
$$

where $D(x)=\prod_{i=1}^{l^{\prime}}\left(x-a_{i}\right)^{r_{i}}$ is the denominator of $\varphi(x)$ with $A^{\prime}=$ $\left\{a_{0}, a_{1}, \cdots, a_{l^{\prime}}\right\}$, and where the $h_{i}(x)$ are polynomials of degree $<r^{\prime}=$ $\sum_{i=0}^{l^{\prime}} r_{i}$. Put $M=\sum_{i=0}^{r^{\prime}-1}\left\|x^{i} / D(x)\right\|_{X}$. If $H_{i}(x)$ is the polynomial obtained from $h_{i}(x)$ by replacing each coefficient of $h_{i}(x)$ with its integral part, it is immediate that $\left\|\left(h_{i}(x)-H_{i}(x)\right) / D(x)\right\|_{X}<M$. Put $p(x)=\sum_{j=m}^{n}\left(H_{i}(x) / D(x)\right) \varphi(x)^{j} ; p(x)$ is an integral $A$-function and

$$
\left\|p(x)-\varphi(x)^{m} h(x)\right\|_{X} \leqq M \sum_{j=m}^{n}\|\varphi(x)\|_{X}^{j}
$$

and hence

$$
\|g(x)-p(x)\|_{X} \leqq \varepsilon+M \cdot\|\varphi(x)\|_{X}^{m} /\left(1-\|\varphi(x)\|_{X}\right) .
$$

Thus if $m$ is sufficiently large, $\|g(x)-p(x)\|_{X}<2 \varepsilon$ and hence $g$ is approximable on $X$. We have just shown that if $g$ vanishes on $K$ then $g$ is approximable on $X$. If $\varepsilon>0$ and $g \in C_{s}(S)$ satisfies $\|g\|_{K}<\varepsilon$, then it is easy to find $g_{1} \in C_{s}(X)$ vanishing on $K$ and satisfying $\left\|g-g_{1}\right\|_{X}<2 \varepsilon$. It is immediate that if $g$ is approximable on $K$ then it is approximable on $X$. Thus we must show that if $g$ is matchable on $J(X, A)$ it is approximable on $K$. By replacing $g$ by $g-p$ where $p$ is an appropriate integral $A$-function, we may assume that $g$ vanishes on $J(X, A)$. Now we must show 
that if $g$ vanishes on $J(X, A)$, then it is approximable on $K$. Choose $\theta \in K-J(X, A)$. Let $\theta=\theta_{1}, \theta_{2}, \cdots, \theta_{m}$ be the conjugates of $\theta$ which are contained in $K$. Since $\theta \notin J(X, A)$, either $\theta$ is not an $A$-integer or $\theta$ has a conjugate outside of $X$. Suppose first that $\theta$ is not an $A$-integer. By Theorem 1.5, the set $\left\{\theta_{1}, \theta_{2}, \cdots, \theta_{m}\right\}$ is $A$-small and hence there exists a normal, integral $A$-function $p$ such that $\left|p\left(\theta_{i}\right)\right|<1$ for $1 \leqq i \leqq m$. Since $\theta$ is not an $A$-integer, none of the $p\left(\theta_{i}\right)$ are 0 . Next suppose that at least one conjugate is outside of $X$. Since $m$ is less than the degree $d$ of $\theta$, there exist, by Minkowski's Theorem on linear forms, integers $b_{0}, b_{1}, \cdots, b_{d-1}$ not all 0 such that $\left|\sum_{j=0}^{d-0} b_{j} \theta_{i}^{j}\right|<1$ for $1 \leqq i \leqq m$. If $p(x)=\sum_{j=0}^{d-1} b_{j} x^{j}$ then $p\left(\theta_{i}\right) \neq 0$, for the degree of $p(x)$ is less than the degree of $\theta$. Thus in either case $p(x)$ is an integral $A$-function with $0<\left|p\left(\theta_{i}\right)\right|<1$ for $1 \leqq i \leqq m$.

By replacing $p$ by $p^{n} h$ where $n$ is a large enough integer and $h$ is an appropriate integral $A$-function, we may assume in addition that $p$ vanishes on all elements of $K$ not conjugate to $\theta$. Let $p_{1}, p_{2}, \cdots, p_{s}$ be the functions obtained for each set of conjugate $A$ integers in $K \sim J(X, A)$. If $n$ is large enough, $\varphi=p_{1}^{n}+p_{2}^{n}+\cdots+p_{s}^{n}$ will satisfy $0<|\varphi(x)|<1$ for $x \in K-J(X, A)$ and $\varphi(x)=0$ for $x \in J(X, A)$. By the earlier part of the proof applied to $K$ instead of $X$, any function in $C_{s}(K)$ which vanishes on $J(X, A)$ is approximable on $K$. By the earlier comments, the proof is complete.

We now give a characterization of $J(X, A)$.

Theorem 4.3. Suppose $X$ is $A^{\prime}$-small for some nonempty $A^{\prime} \subset A$ and that each component of the complement of $X$ contains an element of $A^{\prime}$. There exists an integral A-function $\varphi$ such that $\|\varphi(x)\|<1$ and the zeros of $\varphi$ in $X$ form the set $J(X, A)$.

Proof. Let $q(x)$ be an integral $A$-function whose zeros are the elements of $J(X, A)$. Choose $h \in C_{s}(X)$ satisfying, for all $x \in X:(1)$ $\|h\|_{X}=1 ;(2) h(x)=1$ if $q(x)=0$; (3) $|h(x)|<1 /(2|q(x)|)$ if $|q(x)|>$ $1 / 2 ;(4) h(x) \neq 0$. Such an $h$ is matchable by 1 on $J(X, A)$, hence is approximable on $X$. Any sufficiently good approximation, say, the integral $A$-function $g$, satisfies, for all $x \in X$, (1) $\|g\| \leqq 3 / 2$; (2) $|g(x)|<2 /(3|q(x)|)$ if $|q(x)|>1 / 2$; (3) $g(x) \neq 0$. Put $\phi=g q$ to complete the proof.

\section{REFERENCES}

1. D. G. Cantor, On approximation by polynomials with algebraic integer coefficients, Proceedings of Symposia in Pure Mathematics, Volume XII, Amer. Math. Soc., 1969.

2. M. Fekete, Über die Verteilung der Wurzeln bei gewissen algebraischen Gleichungen mit ganzzahligen Koeffizienten, Math. Z., 17 (1923), 228-249. 
3. M. Febete and G. Szego, On algebraic equations with integral coefficients whose roots belong to a given point set, Math. Z., 63 (1955), 158-172.

4. F. Gantmacher, The Theory of Matrices, Vol. 2, Chelsea, 1959.

5. E. Hewitt and H. Zuckerman, Approximation of polynomials with integral coefficients, a reformulation of the Stone-Weierstrass theorem, Duke Math. J., 26 (1959), $97-116$.

6. S. N. Mergelyan, Uniform approximation to functions of a complex variable, Amer. Math. Soc., Translations, 101 (1954). [Uspehi Mathematiceskih Nauk (N.S.) 7, No. 2 (48), 31-122 (1952).]

7. R. M. Robinson, Intervals containing infinitely many sets of conjugate algebraic integers, Studies in Mathematical Analysis and Related Topics, Stanford University Press, 1962.

8. Conjugate real algebraic integers in point sets, Math. Z., 84 (1964), $415-427$.

9. - Intervals containing infinitely many sets of conjugate algebraic units, Annals of Math., 80, (1964), 411-428.

10. - On the Distribution of Certain Algebraic Integers, Math. Z., 99 (1967), $28-41$.

11. - An extension of Polya's theorem on power series with integer coefficients, Trans. Amer. Math. Soc., 130 (1968), 532-543.

Received September 20, 1974 and in revised form June 29, 1976. This work was supported in part by NSF grant MPS 75-06686.

University of California, Los ANgeles 


\section{PACIFIC JOURNAL OF MATHEMATICS}

\section{EDITORS}

RICHARD ARENS (Managing Editor)

University of California

Los Angeles, California 90024

\section{J. DugunduI}

Department of Mathematics University of Southern California Los Angeles, California 90007

D. Gilbarg and J. Milgram

Stanford University

Stanford, California 94305

\author{
R. A. Beaumont \\ University of Washington \\ Seattle, Washington 98105
}

\section{ASSOCIATE EDITORS}
B. H. NeUmanN
F. WOLF
K. YosHIDA

\section{SUPPORTING INSTITUTIONS}

\author{
UNIVERSITY OF BRITISH COLUMBIA \\ CALIFORNIA INSTITUTE OF TECHNOLOGY \\ UNIVERSITY OF CALIFORNIA \\ MONTANA STATE UNIVERSITY \\ UNIVERSITY OF NEVADA \\ NEW MEXICO STATE UNIVERSITY \\ OREGON STATE UNIVERSITY \\ UNIVERSITY OF OREGON \\ OSAKA UNIVERSITY
}

\author{
UNIVERSITY OF SOUTHERN CALIFORNIA \\ STANFORD UNIVERSITY \\ UNIVERSITY OF HAWAII \\ UNIVERSITY OF TOKYO \\ UNIVERSITY OF UTAH \\ WASHINGTON STATE UNIVERSITY \\ UNIVERSITY OF WASHINGTON \\ AMERICAN MATHEMATICAL SOCIETY
}

The Supporting Institutions listed above contribute to the cost of publication of this Journal, but they are not owners or publishers and have no responsibility for its content or policies.

Mathematical papers intended for publication in the Pacific Journal of Mathematics should be in typed form or offset-reproduced, (not dittoed), double spaced with large margins. Please do not use built up fractions in the text of your manuscript. You may however, use them in the displayed equations. Underline Greek letters in red, German in green, and script in blue. The first paragraph or two must be capable of being used separately as a synopsis of the entire paper. Items of the bibliography should not be cited there unless absolutely necessary, in which case they must be identified by author and Journal, rather than by item number. Manuscripts, in triplicate, may be sent to any one of the editors. Please classify according to the scheme of Math. Reviews, Index to Vol. 39. All other communications should be addressed to the managing editor, or Elaine Barth, University of California, Los Angeles, California, 90024.

The Pacific Journal of Mathematics expects the author's institution to pay page charges, and reserves the right to delay publication for nonpayment of charges in case of financial emergency.

100 reprints are provided free for each article, only if page charges have been substantially paid. Additional copies may be obtained at cost in multiples of 50 .

The Pacific Journal of Mathematics is issued monthly as of January 1966. Regular subscription rate: $\$ 72.00$ a year (6 Vols., 12 issues). Special rate: $\$ 36.00$ a year to individual members of supporting institutions.

Subscriptions, orders for back numbers, and changes of address should be sent to Pacific Journal of Mathematics, 103 Highland Boulevard, Berkeley, California, 94708.

PUBLISHED BY PACIFIC JOURNAL OF MATHEMATICS, A NON-PROFIT CORPORATION

Printed at Kokusai Bunken Insatsusha (International Academic Printing Co., Ltd.), 8-8, 3-chome, Takadanobaba, Shinjuku-ku, Tokyo 160, Japan. 


\section{Pacific Journal of Mathematics}

Patricia Andresen and Marvin David Marcus, Weyl's inequality and

quadratic forms on the Grassmannian .......................

George Bachman and Alan Sultan, Regular lattice measures: mappings and

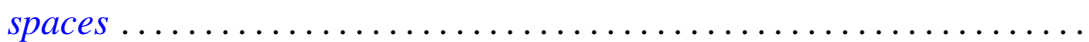

David Geoffrey Cantor, On certain algebraic integers and approximation by rational functions with integral coefficients ...................

James Richard Choike, On the value distribution of functions meromorphic in the unit disk with a spiral asymptotic value ..................

David Earl Dobbs, Divided rings and going-down................ 353

Mark Finkelstein and Robert James Whitley, Integrals of continuous

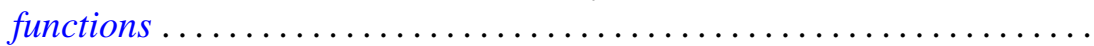

Ronald Owen Fulp and Joe Alton Marlin, Integrals of foliations on manifolds with a generalized symplectic structure ...............

Cheong Seng Hoo, Principal and induced fibrations .................

Wu-Chung Hsiang and Richard W. Sharpe, Parametrized surgery and

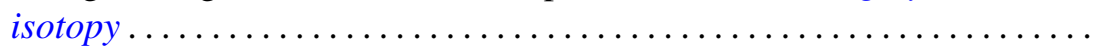

Surender Kumar Jain, Surjeet Singh and Robin Gregory Symonds, Rings whose proper cyclic modules are quasi-injective .................

Pushpa Juneja, On extreme points of the joint numerical range of commuting normal operators...

Athanassios G. Kartsatos, Nth order oscillations with middle terms of order $N-2$.

John Keith Luedeman, The generalized translational hull of a

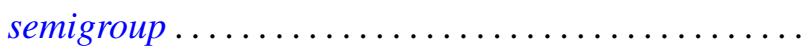

Louis Jackson Ratliff, Jr., The altitude formula and DVR's ...

Ralph Gordon Stanton, C. Sudler and Hugh C. Williams, An upper bound for the period of the simple continued fraction for $\sqrt{D}$...

David Westreich, Global analysis and periodic solutions of second order systems of nonlinear differential equations...

David Lee Armacost, Correction to: "Compactly cogenerated LCA

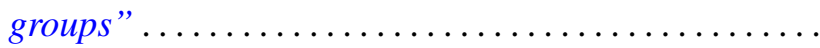

Jerry Malzan, Corrections to: "On groups with a single involution" .

David Westreich, Correction to: "Bifurcation of operator equations with unbounded linearized part" ...................... 